\title{
PARTNER cohort A-TAVR is an option for operable severe aortic stenosis
}

The results of the cohort A analysis from the PARTNER trial, which were first presented at the ACC Scientific Sessions in April 2011, have now been published in the New England Journal of Medicine. Transcatheter aortic valve replacement (TAVR) was found to be noninferior to surgical valve replacement in terms of 1-year all-cause mortality among 699 high-risk patients with severe, symptomatic aortic stenosis.

“Catheter-based 'percutaneous' techniques for valve replacement started gaining attention about 10 years ago, based on animal experimentation and a first-in-man experience in France by Alain Cribier," says Dr Craig Smith, Surgeon-in-Chief at New York-Presbyterian Hospital and Columbia University Medical Center, NY, USA, who was lead author of the cohort A analysis. "The paradigm change this implied was electrifying to anyone involved in the aggressive treatment of valve disease."

The data from cohort A follow the publication in September 2010 of the groundbreaking results from cohort $B$ of the PARTNER trial, and strengthen the evidence base for the use of TAVR in patients with severe aortic stenosis. Cohort B showed us that TAVR was superior to standard therapy in patients for whom surgery was not an option; now, this new analysis confirms that the transcatheter strategy is a feasible alternative to surgery in high-risk, operable patients as well.

Patients with severe aortic stenosis - aortic valve area $<0.8 \mathrm{~cm}^{2}$, and either a mean aortic valve gradient $\geq 40 \mathrm{mmHg}$ or a peak aortic jet velocity $\geq 4.0 \mathrm{~m} / \mathrm{s}$ - and NYHA class II-IV symptoms were enrolled from 25 centers in the USA,

Canada, and Germany. Participants were randomly assigned to undergo surgical aortic valve replacement $(n=351)$ or to TAVR $(n=348)$ with the Edwards SAPIEN ${ }^{\circledR}$ heart-valve system (Edwards Lifesciences Corporation, Irvine, CA, USA). In the TAVR group, transfemoral access was used in 244 patients and transapical access in 104 patients. Neither the mean age of patients ( 84 years) nor the mean Society of Thoracic Surgeons risk score $(\sim 11.8)$ differed significantly between the two treatment groups. At 30 days after the procedure, all-cause mortality was $3.4 \%$ in the TAVR group and $6.5 \%$ in the surgical group $(P=0.07)$, rising to $24.2 \%$ and $26.8 \%$, respectively, after 1 year $(P=0.44)$. However, $\mathrm{Dr}$ Smith points out that "in the transfemoral group, 30-day periprocedural mortality was the best ever reported $3.7 \%$ in the as-treated analysis, $3.3 \%$ by intention-to-treat) and approaches superiority compared with surgery".

Both procedures had their drawbacks; patients who underwent surgery were more likely to experience new-onset atrial fibrillation (16.0\% versus $8.6 \%$, $P=0.006)$ and major bleeding $(19.5 \%$ versus $9.3 \%, P<0.001)$ than those in the TAVR group, whereas TAVR was associated with an increased risk of major vascular complications at 30 days $(11.0 \%$ versus $3.2 \%$ for surgery, $P<0.001)$. In addition, a moderately significant increase in neurological events was reported in the TAVR group at 30 days (5.5\% versus $2.4 \%, P=0.04)$ and 1 year $(8.3 \%$ versus $4.3 \%, P=0.04)$.

"Going forward, TAVR is likely to be approved by the FDA for inoperable patients, and a new randomized trial will begin soon comparing TAVR with [surgical] aortic valve replacement in lower-risk patients," concludes Dr Smith. "How far down the risk spectrum the trial will move is not yet known with any certainty".

Alexandra King

Original article Smith, C. R. et al. Transcatheter versus surgical aortic valve replacement in high-risk patients. N. Engl. J. Med. 364, 2187-2198 (2011) 